\title{
Formation of Moments Influence Matrix in Frame Based on Graph of its Design Scheme
}

\author{
V.A. Monakhov ${ }^{1, ~}{ }^{*}$ and A.I. Shein ${ }^{1}$ \\ ${ }^{1}$ Penza State University of Architecture and Construction, Penza, Russia)
}

\begin{abstract}
A procedure is proposed for the automated construction of the bending moments influence matrix in the frame based on the graph of its discrete model. Forming the incidence matrix of the graph characterizing the topological structure of the design scheme of the frame, through matrix transformations of the displacement vectors, at the transition from local coordinate systems to a global system, it is possible to establish the relationship between the nodal displacements and the displacement increments of individual sections in the direction of the axis of the segment and perpendicular to it. The composition of only three initial matrices of the frame structure, the incidence matrix of the graph, the node coordinate matrix and the matrix of the frame model internal rigidity, solves the problem of automatic formation of the bending moments influence matrix with the help of a PC. The procedure proposed for the construction of the influence matrix, makes it possible to find the forces in the frame structure caused by external load, in the matrix form.
\end{abstract}

During the intensive development of the finite element method in the second half of the $20^{\text {th }}$ century, a number of Russian scientists in the field of structural theory attempted to create alternative numerical methods to calculate frame structures with the help of computers [1] [4]. In particular, A.R. Rzhanitsyn proposed to analyze the stress-strain state of frames in matrix form based on the duality principle of problems in structural theory. Representing any elastic frame structure in the form of a connected set of bar segments obtained by discretizing the system, a geometric [1] (kinematic [3]) matrix characterizing the deformed state of the structure, was taken as a key for solving the problem. In accordance with the duality principle, the stress state of the system in this case is determined by the equilibrium matrix, which is found by transposing the geometric matrix. As a rule, these authors limited themselves to the consideration of the bending deformations of frames, although the regard of the longitudinal deformations of the bars, as an example, presents no particular difficulties [4].

At that time, to create a fully automated method for the formation of a geometric matrix, it was sufficient to provide it with a description of the frame structure, its geometric parameters, as well as physical and mechanical properties, in matrix form. This description is completed in this work. As a result, the point at issue is the development of an automated method for frame structure calculation, which is an alternative to the finite element method.

*Corresponding author: monahovnn@mail.ru 
Further on, according to the design scheme of the frame (Fig. 1, a), which was taken into consideration in article [1], along with the basic operations of the numerical method, including initial steps associated with the specification of the frame design scheme, structure and physical and mechanical properties in digital form, a calculation algorithm is presented, the ultimate goal of which is the formation of the frame bending moments influence matrix $[\Lambda]$. The diagram of bending moments in this case can be built on the basis of the moment matrix $[M]=[\Lambda] \bar{P}$, where $\bar{P}=\left(\xi_{1} p, \xi_{2} p, M\right)^{T}$ - external action column vector. The columns of the matrix $[M]$ are the values of the bending moments corresponding to the loads that are determined by the components of vector $\bar{P}$.

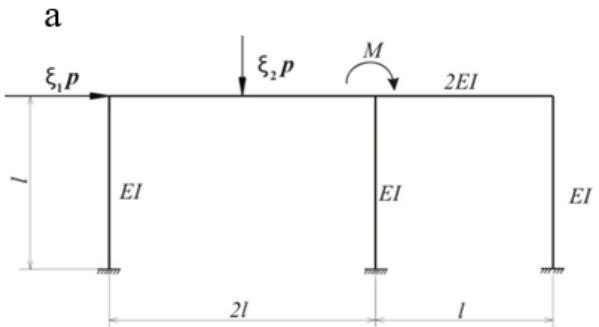

$\mathrm{c}$

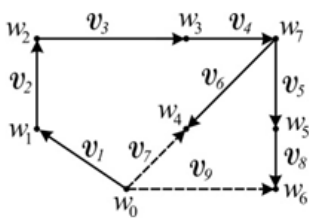

$\mathrm{b}$

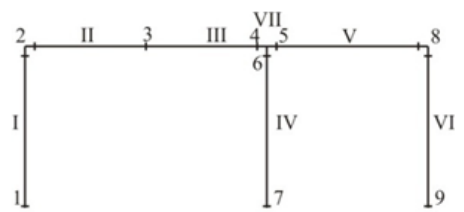

d

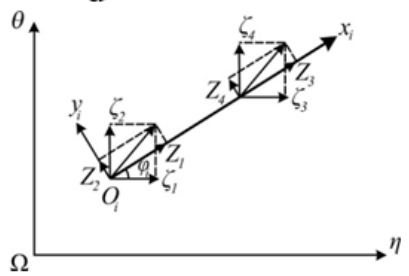

Fig. 1. Design scheme of the frame, its model, graph and indexing of nodal displacements

Referring to the discrete model of the frame (Fig. 1, b) and using the methods of graph theory, the topology of the frame structure can be described using the model graph (Fig. 1, c) and the accompanying incidence matrix [5], [6]:

$$
[S]_{(6 \times 9)}=\left[\begin{array}{ccccccccc}
-1 & 1 & 0 & 0 & 0 & 0 & 0 & 0 & 0 \\
0 & -1 & 1 & 0 & 0 & 0 & 0 & 0 & 0 \\
0 & 0 & -1 & 1 & 0 & 0 & 0 & 0 & 0 \\
0 & 0 & 0 & -1 & 1 & 1 & 0 & 0 & 0 \\
0 & 0 & 0 & 0 & -1 & 0 & 0 & 0 & 1 \\
0 & 0 & 0 & 0 & 0 & -1 & 1 & 0 & 0
\end{array}\right] .
$$

Finitely, before this, when digitizing the frame, for example, into 6 sections (links), the segments, as well as the division points, are numbered. The base of the frame is assigned number "0" and only then the graph is built. The points of this graph $w_{j}(j=I, I I, \ldots, V I)$ are associated with individual parts of the frame, and its sides ("arcs" $u_{i}(i=1,2, \ldots, 9)$ ) are associated with design sections (nodes) (Fig. 1, c). The degree of digitizing depends on a number of circumstances - the load nature, the difference in transverse dimensions and the type of material of bars in different parts of the frame, the power of the computer, etc. 
Introducing the matrix of coordinates of model nodes in the global coordinate system $\eta \Omega \theta$

$$
[K]=\left[\begin{array}{cccc}
\eta_{1} & \eta_{2} & \cdots & \eta_{9} \\
\theta_{1} & \theta_{2} & \cdots & \theta_{9}
\end{array}\right]_{(2 \times 9)}
$$

where the first row of the matrix contains the abscissas of the coordinates of the nodes, and the second - their ordinates, and calculating the matrix product

$$
[U]=[K][S]^{T},
$$

a matrix of projections of bar segments on the axis of the global coordinate system is determined. Directly the lengths of the elements of the frame structure are calculated by the following formula $[L]=\sqrt[*]{\sum([U])^{* 2}}$.

In this expression, the symbol $(\ldots)^{* 2}$ means squaring each element of the matrix $[U]$, followed by summing the squares of the elements by columns and extracting the roots from the sums obtained. After the determination of the lengths of the segments and formation of a diagonal matrix of lengths

$$
[L]=\left[\begin{array}{llll}
l_{1} & & & \\
& l_{2} & & \\
& & \ddots & \\
& & & l_{6}
\end{array}\right]=\left[\begin{array}{llll}
1 & & & \\
& 1 & & \\
& & \ddots & \\
& & & 1
\end{array}\right] \cdot l,
$$

it is easy to calculate the vector of direction cosines of segments in accordance with the following formula $\bar{N}_{\eta}=[L]^{-1} \bar{U}_{\eta}$, where $\bar{U}_{\eta}$ - top row of the matrix $[U]$ (for more details, see [7], [8]). The latter serves as the basis for the formation of the frame sections tilt matrices

$$
\left[\phi_{j}\right]=\left[\begin{array}{cc}
\cos \varphi_{j} & \sin \varphi_{j} \\
-\sin \varphi_{j} & \cos \varphi_{j}
\end{array}\right] \quad(j=1,2, \ldots, 6) .
$$

Thus, to describe the geometric scheme of the frame structure, only two matrices are sufficient - the node coordinate matrix and the incidence matrix.

The physical and mechanical properties of frame elements can also be described via matrices. If bending deformations prevail in the system, then the stiffness cell (matrix) of any bar segment has the following form

$$
\left[c_{j}\right]=\left[\begin{array}{ll}
4 & 2 \\
2 & 4
\end{array}\right] \frac{E_{j} I_{j}}{l_{j}} \quad(j=1,2, \ldots, 6) .
$$

Here, $E_{j} I_{j}(j=1,2, \ldots, 6)$ - bending stiffness of the $j$ th frame segment. The set of such cells of the complete set of elements forms a quasi-diagonal internal stiffness matrix of the entire frame

$$
[C]=\left[\begin{array}{llll}
{\left[c_{1}\right]} & & & \\
& {\left[c_{2}\right]} & & \\
& & \ddots & \\
& & & {\left[c_{j}\right]}
\end{array}\right] .
$$

In the process of further calculations, the stiffness of the cells of all elements $\left[c_{j}\right]$ reduces to the stiffness of any of such elements. 
The graph incidence matrix $[S]$, along with the node coordinate matrix $[K]$ and internal stiffness matrix $[C]$, serves as the basis for the development of an algorithm for calculating frame structures, which is presented below. The ultimate goal of the algorithm is to form a bending moments influence matrix $[\Lambda]$ in the frame. In contrast to $[1]$, in this article the calculation process is proposed to be totally formalized so that all calculations can be performed automatically.

The first step of the algorithm for the numerical calculation of the frame is to form the quartic block matrices

$$
\left[\Phi_{i}\right]=\left[\begin{array}{ccc}
{\left[\phi_{i}\right]} & \vdots & 0 \\
\cdots & \vdots & \cdots \\
0 & \vdots & {\left[\phi_{i}\right]}
\end{array}\right],
$$

with the help of which the transition from global displacements of nodes $\bar{\zeta}^{(i)}(i=1,2, \ldots, 6)$ of a separate section to local ones $\bar{Z}^{(i)}(i=1,2, \ldots, 6)$ is carried out according to the matrix transformation $\bar{Z}^{(i)}=\left[\Phi_{i}\right] \bar{\zeta}^{(i)}(i=1,2, \ldots, 6)$ (Fig. 1, d). A set of similar transformations for all the frame elements is represented by the following matrix

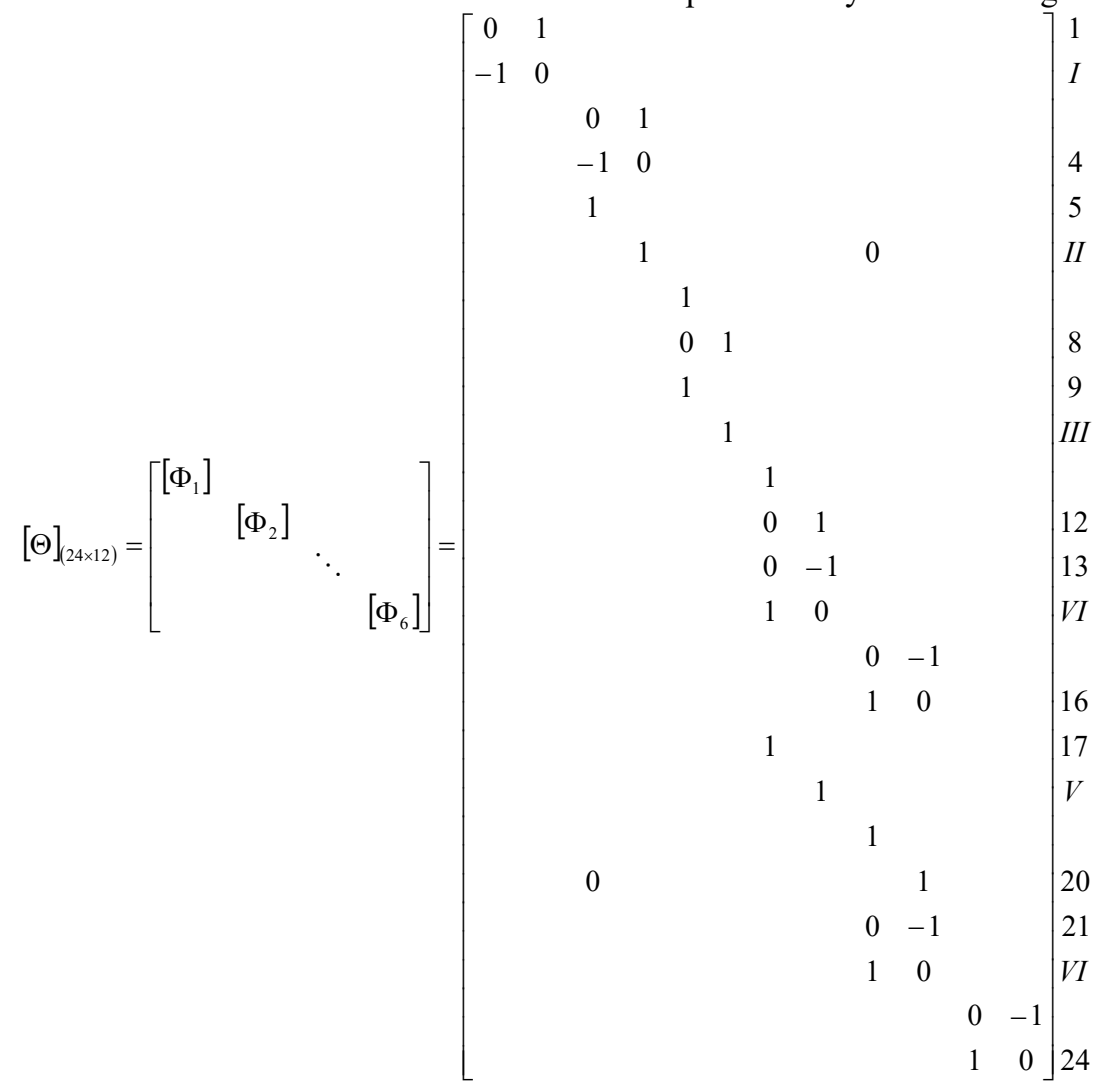

which makes it possible to establish connection between local and global displacements of the nodes of the entire frame in matrix form $\bar{Z}=[\Theta] \bar{\zeta}$. The number of rows of this matrix is determined by the order of the vector of nodal displacements $\bar{\zeta}$ in the global coordinate system $\eta \Omega \theta$, the number of columns is determined by the number of independent nodal displacements of the frame that make up the vector of local variables $\bar{Z}$ 
(Fig. 1, d). The numbers outside the bracket of the matrix $[\Theta]$ are noteworthy. The elements of the matrix located within the rows with Arabic numerals refer to the sections of the frame indicated in Figure 1, b by the Roman ones. Connection of increments of nodal displacements directly with displacements of nodes in the global system $\bar{\gamma}_{(12)}=[\Gamma]_{(12 \times 14)} \bar{\zeta}_{(14)}$ is defined by the following matrix transformation $[\Gamma]_{(12 \times 14)}=[\widetilde{S}]_{(12 \times 24)}[\Psi]_{(24 \times 14)}$. Here, $\bar{\gamma}_{(12)}=\left(\lambda_{1}, \chi_{1}, \lambda_{2}, \chi_{2}, \ldots, \lambda_{6}, \chi_{6}\right)$ - column vector of nodal increments of displacements in the direction of the segment axis (absolute elongations $\lambda_{j}(j=1,2, \ldots, 6)$ ) and increments of displacements of the ends of the segments in the direction perpendicular to the axis (the so-called shifts $\chi_{j}(j=1,2, \ldots, 6)$ ), correspondingly; $[\widetilde{S}]_{(12 \times 24)}$ - expanded incidence matrix of the frame structure graph, formed on the basis of the incidence matrix $[S]$ via its expansion [6]. If, when calculating the frame, it is decided to neglect the longitudinal deformations of the bars when the frame is deformed, then the matrix $[\Gamma]_{(12 \times 14)}$ must be corrected by deleting odd lines in it. The deletion of the indicated lines results in the following matrix

$$
\left[G_{0}\right]_{(6 \times 14)}=\left[\begin{array}{cccccccccccccc}
1 & 0 & -1 & 0 & 0 & 0 & 0 & 0 & 0 & 0 & 0 & 0 & 0 & 0 \\
0 & 0 & 0 & -1 & 0 & 1 & 0 & 0 & 0 & 0 & 0 & 0 & 0 & 0 \\
0 & 0 & 0 & 0 & 0 & -1 & 0 & 1 & 0 & 0 & 0 & 0 & 0 & 0 \\
0 & 0 & 0 & 0 & 0 & 0 & -1 & 0 & 1 & 0 & 0 & 0 & 0 & 0 \\
0 & 0 & 0 & 0 & 0 & 0 & 0 & -1 & 0 & 0 & 0 & 1 & 0 & 0 \\
0 & 0 & 0 & 0 & 0 & 0 & 0 & 0 & 0 & 0 & -1 & 0 & 1 & 0
\end{array}\right] .
$$

Taking into account the immobility of the supports $\left(\zeta_{1}=\zeta_{2}=\zeta_{9}=\zeta_{10}=\zeta_{13}=\zeta_{14}=0\right)$, in matrix $\left[G_{0}\right]_{(6 \times 14)}$ it is necessary remove the two outermost columns on each side and two more - the ninth and the tenth - in the middle; in addition, to remove the fifth column due to the continuity of the crossbar in the third section. This results in the rectangular matrix

$$
[G]_{(6 \times 7)}=\left[\begin{array}{ccccccc}
-1 & 0 & 0 & 0 & 0 & 0 & 0 \\
0 & -1 & 1 & 0 & 0 & 0 & 0 \\
0 & 0 & -1 & 0 & 1 & 0 & 0 \\
0 & 0 & 0 & -1 & 0 & 0 & 0 \\
0 & 0 & 0 & 0 & -1 & 0 & 1 \\
0 & 0 & 0 & 0 & 0 & -1 & 0
\end{array}\right] .
$$

Using it, the shifts of the bars are calculated depending on the nodal displacements $\bar{\chi}=[G]_{(6 \times 7)} \bar{\zeta} ;$ now, it is expedient to renumber the vector components $\bar{\zeta}=\left(\zeta_{3}, \zeta_{4}, \zeta_{6}, \zeta_{7}, \zeta_{8}, \zeta_{11}, \zeta_{12}\right)$ from one to seven, setting $\bar{\zeta}_{1}=\bar{\zeta}_{3}, \bar{\zeta}_{2}=\bar{\zeta}_{4}, \bar{\zeta}_{3}=\bar{\zeta}_{6}, \bar{\zeta}_{4}=\bar{\zeta}_{7}, \bar{\zeta}_{5}=\bar{\zeta}_{8}, \bar{\zeta}_{6}=\bar{\zeta}_{11}, \bar{\zeta}_{7}=\bar{\zeta}_{12}$

As a result of the multiplication of matrix $[G]_{(6 \times 7)}$ by transposed incidence matrix $[S]_{(9 \times 6)}^{T}$, a complete geometric matrix is formed with the following structure 


$$
\left[H_{0}\right]_{(9 \times 7)}=\left[\begin{array}{ccccccc}
1 & 0 & 0 & 0 & 0 & 0 & 0 \\
-1 & 1 & -1 & 0 & 0 & 0 & 0 \\
0 & -1 & 2 & 0 & -1 & 0 & 0 \\
0 & 0 & -1 & 0 & 1 & 0 & 0 \\
0 & 0 & 0 & 0 & 1 & 0 & -1 \\
0 & 0 & 0 & 1 & 0 & 0 & 0 \\
0 & 0 & 0 & -1 & 0 & 0 & 0 \\
0 & 0 & 0 & 0 & -1 & 1 & 1 \\
0 & 0 & 0 & 0 & 0 & -1 & 0
\end{array}\right] .
$$

Since the axial deformations of the crossbar are negligible, then $\zeta_{3}=\zeta_{7}=\zeta_{11}$, which makes it possible to combine the $4^{\text {th }}$ and $6^{\text {th }}$ columns with the $1^{\text {st }}$ one. Also ignoring the longitudinal deformations in the legs, columns 2, 5 and 7 should be deleted from the matrix. As a result, this matrix is reduced to two columns. Adding to it the third column, corresponding to the rotation operation of the middle node by an angle $\varphi$, a geometric matrix is formed in the final form (compare with [1])

$$
[H]_{(9 \times 3)}=\left[\begin{array}{rrr}
1 & 0 & 0 \\
-1 & -1 & 0 \\
0 & 2 & 0 \\
0 & -1 & 1 \\
0 & 0 & -1 \\
1 & 0 & -1 \\
-1 & 0 & 0 \\
1 & 0 & 0 \\
-1 & 0 & 0
\end{array}\right] .
$$

Owing to matrix $[H]$, the displacement vector $\bar{Y}_{(3)}=\left(\zeta_{1}, \zeta_{2}, \varphi\right)$ is transformed during the transition to bending deformations characterized by the vector $\bar{x}_{(9)}=[H]_{(9 \times 3)} \bar{Y}_{(3)}$. In the case under consideration, bending deformations are concentrated at the discretization points of the frame. The components of the obtained vector are the angles of mutual rotation $x_{(i)}(i=1,2, \ldots, 9)$ of the adjacent segments of the bars at the frame nodes, including the support ones (Fig. 2). When forming the geometric matrix of the frame under consideration, it is necessary to pay attention to the peculiarity that due to the presence of an independent parameter of the deformed state of the frame, namely, the angle of rotation of the middle node by the angle $\zeta_{25} \equiv \varphi$, which corresponds to the deformation pattern caused by the rotation of the node (Fig. 2, b). It should be remembered that the angular displacement index $\zeta_{25}$ corresponds to the initial numbering of independent displacements of the problem under consideration in the global coordinate system. Due to the reduction in the number of components of the global displacement vector, at this stage, one more reindexing is performed, namely, now $\zeta_{1}=\zeta_{3}, \zeta_{2}=\zeta_{4}, \zeta_{3}=\varphi$. The new numbering of displacements corresponds to the indexing of external influences shown in Fig. 2. 

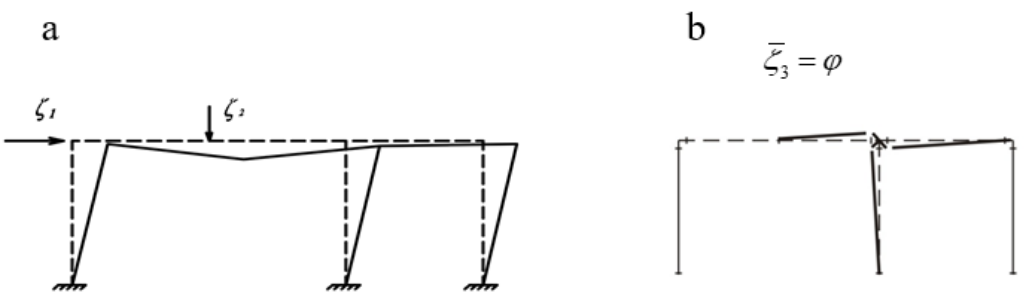

Fig. 2. Schemes of concentrated bending deformations of the frame

All operations concerning the matrix compilation can be fully automated and performed on a PC. The construction of the frame graph and the accompanying incidence matrix is also performed automatically [9].

The values of the bending moments in the design sections are found by the following formula

$$
\bar{M}=[\Lambda] \bar{P},
$$

where $[\Lambda]=[C][H][R]^{-1}$ - moments influence matrix, $[R]=[H]^{T}[C][H]$ - frame external stiffness matrix, $\bar{P}=\left(p_{1}, p_{2}, M\right)^{T}-$ column vector of external concentrated loads. Determination of stiffness matrix $[R]$ is advisable to be performed in advance. To do this, first it is necessary to find the matrix of unit efforts in the design sections of the frame

$$
\begin{aligned}
& {[T]_{(9 \times 3)}=[C]_{(9 \times 9)}[H]_{(9 \times 3)}=\left[\begin{array}{ccccccccc}
2 & -1 & & & & & & & \\
-1 & 4 & -1 & & & & & & \\
& -1 & 4 & -1 & 0 & 0 & 0 & 0 & 0 \\
& & -1 & 2 & & & & & \\
& & & & 2 & & & -1 & \\
0 & 0 & 0 & 0 & 0 & 2 & -1 & 0 & \\
0 & 0 & 0 & 0 & 0 & -1 & 2 & 0 & \\
& & & & -1 & 0 & 0 & 2 & -1 \\
0 & 2 & 0 \\
-1 & -1 & 1 \\
0 & 0 & -1 \\
1 & 0 & -1 \\
-1 & 0 & 0 \\
1 & 0 & 0 \\
-1 & 0 & 0
\end{array}\right] \frac{2 E I}{l}=} \\
& =\left[\begin{array}{rrr}
3 & 1 & 0 \\
-5 & -6 & 0 \\
1 & 10 & -1 \\
0 & -4 & 2 \\
-1 & 0 & -2 \\
3 & 0 & -2 \\
-3 & 0 & 1 \\
5 & 0 & 1 \\
-3 & 0 & 0
\end{array}\right]
\end{aligned}
$$

Then the result obtained should be multiplied by the transposed matrix $[H]^{T}$. As a result, the external stiffness matrix takes the following form 


$$
[R]_{(3 \times 3)}=[H]_{(3 \times 9)}^{T}[T]_{(9 \times 3)}=\left[\begin{array}{ccc}
22 & 7 & -2 \\
7 & 30 & -4 \\
-2 & -4 & 6
\end{array}\right] .
$$

The matrix of bending moments in the design sections of the frame is equal to

$$
[M]_{(9 \times 3)}=[T]_{(9 \times 3)}[R]_{(3 \times 3)}^{-1} \bar{P}_{(3)}=[\Lambda]_{(9 \times 3)} \bar{P}_{(3)}=\left[\begin{array}{rrr}
0,1385 & -0,0079 & 0,0514 \\
-0,1863 & 0,1809 & -0,1827 \\
-0,0629 & 0,3545 & 0,0486 \\
0,0605 & -0,1101 & 0,2801 \\
-0,0690 & -0,0345 & -0,3793 \\
0,1295 & 0,0756 & -0,3406 \\
-0,1391 & -0,0532 & 0,1557 \\
0,2597 & 0,0290 & 0,2332 \\
-0,1488 & -0,0309 & -0,0290
\end{array}\right] \cdot\left(\begin{array}{c}
\xi_{1} p \\
\xi_{2} p \\
M
\end{array}\right) .
$$

The matrix columns $[\Lambda]_{(9 \times 3)}=[T]_{(9 \times 3)}[\Delta]_{(3 \times 3)}$, where $[\Delta]_{((\times 3)}=[R]_{(3 \times 3)}^{-1}$ - external frame compliance matrix, characterize the values of bending moments in the design sections of the frame arising from single external influences in the direction of the independent nodal displacements taken in the calculation (Fig. 3, a, b, c).

a)

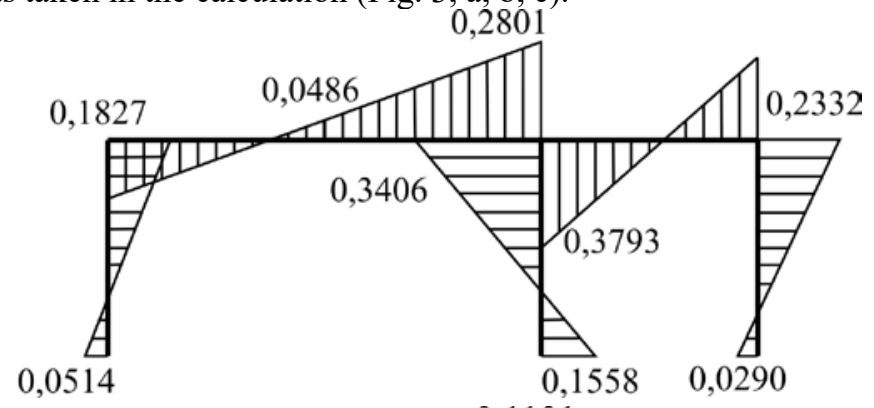

b)

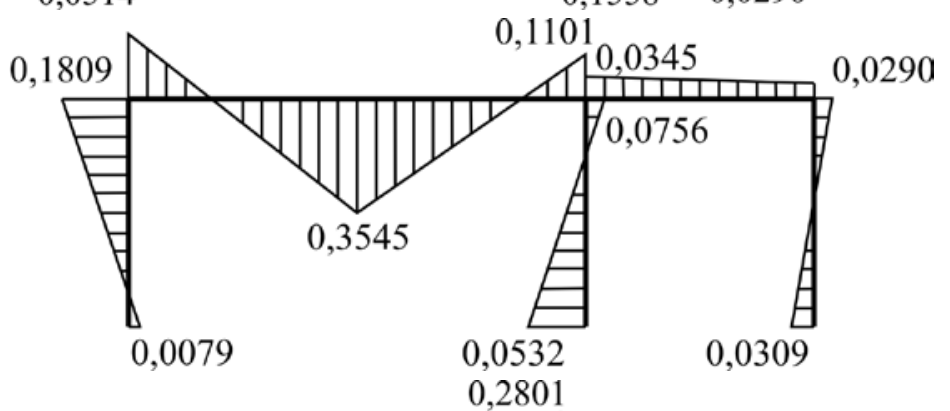

c)

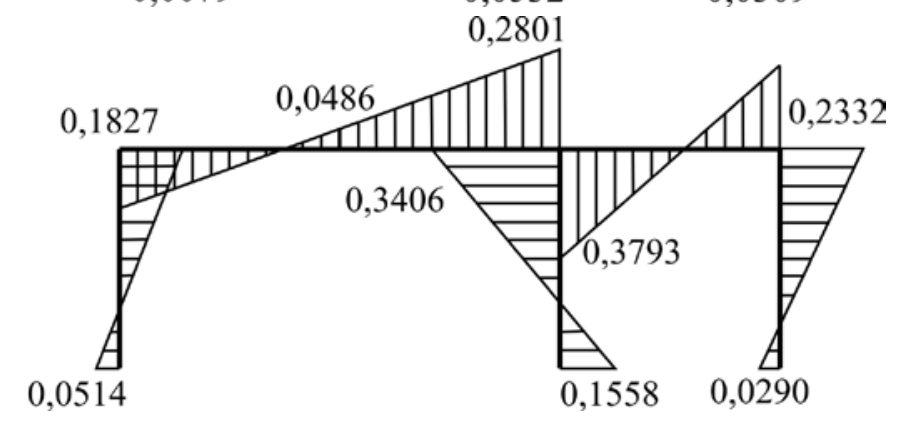

Fig. 3. Bending moment diagrams in the frame 


\section{Conclusion}

The application of geometric matrix $[H]$, which is formed on the basis of the graph of the frame design scheme, makes it possible to form the bending moments influence matrix and, thereby, perform the frame structure calculations in the automatic mode. The proposed calculation method can be considered as an alternative to the finite element method.

\section{References}

1. Rzhanitsyn, A.F. Calculation of Frame Structures with the Help of Duality Principle // Research on Theory of Structures. Issue XXIV. - 1980. - P. 10-23.

2. Maslennikov, A.M. Calculation of Statically Indeterminate Systems by the Displacement Method in Matrix Form [Text] A.M. Maslennikov / - M.: Stroyizdat, 1970. - $128 \mathrm{p}$.

3. Protsenko, A.M. Calculation of Elastoplastic Frame Structures [Text] A.M. Protsenko / - M.: Stroyizdat, - 1978. - 288 p.

4. Smirnov, A.F. Methods for Calculating Frame Structures, Plates and Shells with the Help of Computer [Text] Edited by A.F. Smirnov. Part 1. / - M.: Stroyizdat, - 1976. $451 \mathrm{p}$.

5. Harary, A. Graph Theory [Text] A. Harary / - M.: Nauka, - 2003. - 322 p.

6. Perelmuter, A.V. On Application of Graph Theory to Some Problems of Structural Mechanics // Structural Mechanics and Calculation of Structures. - 1965. - No. 3. - P. 6-13.

7. Reksha, V.V. Application of Graph Theory in Matrix Form of Displacement Method // Structural Mechanics and Calculation of Structures. - 1978. - No. 1. - P. 33-35.

8. Spodaryev, Yu.P. Matrix Method for Calculating Statically Definable Structures // Structural Mechanics and Calculation of Structures. - 1975. - No. 3. - P. 13-18.

9. Zubov, V.S. Programmer's Reference. Basic Methods for Solving Graph Problems and Sorting [Text] V.S. Zubov. / - M.: Filin Publishing House, 1999. - 256 p. 